\title{
Complex Eigenvalue Analysis and Parameters Analysis to investigate the formation of railhead corrugation in short radius curves
}

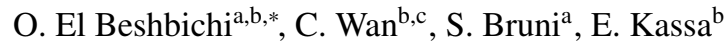 \\ ${ }^{a}$ Politecnico di Milano, Dipartimento di Meccanica, Via la Masa 1 I-20156 Milano, Italy \\ ${ }^{b}$ Norwegian University of Science and Technology NTNU, NO-7491 Trondheim, Norway \\ ${ }^{c}$ Rambøll, 7042 Trondheim, Norway
}

\begin{abstract}
Rail corrugation, a quasi-sinusoidal irregularity of the rail head, is a common issue experienced throughout the railway networks worldwide. It generally leads to high wheel-rail dynamic loads, increased noise emission, and poor ride comfort. Most commonly, rail corrugation is likely to develop on short radius curves. This paper aims to study the numerical feasibility of the prediction of self-excited vibrations for the study of rutting corrugation formation without excitation from initial rail roughness. A finite element model for the prediction of the self-excited vibrations of the leading wheelset-rails system in a short radius curve has been developed in ABAQUS. The friction coupling between the wheel and rail is taken into account. It is assumed that the lateral creep forces between wheel and rail are quasi-saturated. The proposed model is applied to investigate the effect of several structural factors on self-excited vibrations occurrence. Results show a strong match between the typical wavelength of rutting corrugation and the numerical results obtained in this paper, similarities with experimental evidence on rutting corrugation, and a strong influence of the interaction effect of the wheelset cross-section and the track gauge on self-excited vibrations occurrence. For a typical European wheelset cross-section, self-excited vibrations occurred only for a wider track gauge, while for the considered Chinese wheelset cross-section, self-excited vibrations occurred only for a standard track gauge. Therefore, according to the method employed, wheelset cross-section might be an inhibitor factor at a particular track gauge. A parameter sensitivity analysis shows that the friction coefficient is correlated linearly with the system instability and the frequency of the unstable modes of vibration.
\end{abstract}

Keywords: Rutting corrugation, self-excited vibrations, complex eigenvalue analysis, creep forces saturation, wheelset-track system.

\section{Introduction}

Corrugations are a type of quasi-sinusoidal irregularities developing in both rails and wheels. The term is generally used with specific reference to short-wavelength irregularities. The classification used by Alias [1] considers corrugations as irregularities a wavelength between 30 and $80 \mathrm{~mm}$, in contrast to short waves with a wavelength between 150 and $300 \mathrm{~mm}$, and long waves with a wavelength up to $2000 \mathrm{~mm}$. Rail corrugation has been constituting a serious issue ever since rail transportation is utilised as a transportation means. In the 1930s, up to $46 \%$ of the German Railway network was seriously corrugated [2]. Rail corrugation gives rise to poor comfort, dynamic loading of both vehicle and track components, and high levels of noise. Measurements by Vadillo et al. [3] indicated that even in its initial phase and almost invisible, corrugation raised noise emission levels by $6 d B(A)$. Despite of the extensive research on the topic, rail corrugation continues to be a problem whose principal means of control is grinding of rail. However, grinding is rather an expensive measure which is palliative than a solution since corrugation eventually develops. In the 1980s, costs for

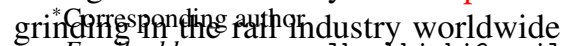

Email address: omar.elbeshbichi@gmail.com (O. El Beshbichi) were in the order of at least US $\$ 10^{8}$ per annum [4]. It was reported that corrugation typically occurs primarily on the low rail in short radius curves (usually curves of less than $450 \mathrm{~m}$ radius) and to a lesser extent in tangent ${ }^{\text {ial }}$ tracks at traction or braking sites [4].

Rail corrugation formation mechanisms are presented by S.L. Grassie and J. Kalousek in their review [5], which is considered to be the most complete work on the subject, later revisited by Grassie himself [6]. The two corrugation formation mechanisms are composed of a wavelength fixing mechanism and a damage mechanism. The wavelength fixing mechanism repre-sents the vibration behavior of the system in terms of vibration modes whose vibration frequency is directly related to the cor-rugation formation wavelength. The damage mechanism is the rail material removal mechanism. The most common damage mechanism is wear, even though several other mechanisms can be found, i.e., plastic flow and plastic bending. The frequencies associated with the wavelength fixing mechanisms are directly related to the corrugation wavelength on account of the above-mentioned wear mechanism. This relation can be expressed as [6]:

$$
\lambda=\frac{v}{f}
$$

where $\lambda$ is the corrugation wavelength, $v$ is the speed of those 
trains that give rise to the corrugation and $f$ the frequency of the pertinent wavelength fixing mechanism.

Among the many known rail corrugation morphologies, the most common ones are the pinned-pinned resonance corrugation, P2 resonance corrugation, and rutting corrugation [5]. They are described by means of their wavelength fixing mechanism. The pinned-pinned resonance corrugation is characterized by the vertical vibration of the rails as if they were pinned at the sleep-ers and is related to frequencies around 800 $1200 \mathrm{~Hz}$. P2 resonance corrugation is associated with the vibration of the unsprung mass on the track stiffness and is related to frequencies between 50 and $100 \mathrm{~Hz}$. These corrugation types are commonly found in straight lines.

On the other hand, rutting corrugation is associated to torsional/bending vi-brations of the leading wheelsets caused by roll-slip vibrations related to the saturation of the traction forces, i.e., where the traction ratio $(\mathrm{T} / \mathrm{N})$ is close to the friction limit and is related to frequencies between 250 and $400 \mathrm{~Hz}$. T is the tangential contact force while $\mathbf{N}$ is the normal contact force at the wheel-rail contact. In fact, contact forces in the leading wheelset are greatest. Rutting corrugation is typi-cal on short radius curves, even though it can also be found in straight lines when the traction or braking forces are sufficiently high. It appears most commonly on the low rail. 'Rutting' has been defined as a specific type of corrugation by S. L. Grassie [5]. Discrete irregularities such as welds and joints are com-mon 'triggers' for rutting corrugation, and often fix the position of corrugation along the rail. Usually, the wheel on the leading wheelset in a bogie is involved in the formation of rutting corrugation because of the high tangential force from curving. The damage mechanism for rutting is most commonly wear [6]. The effect of lateral creep $\mathrm{age}$ force on rail corrugation on the low rail at sharp curves has been studied by Ishida et al. [7]. Their experimental investigations showed the prime relevance of lateral creep age forces and rail joints on the formation of rutting corrugation, and roll-slip between rail and wheel as a possible physical cause. A non-linear time domain model of a bogie cornering with a modal description of the wheelset has been used for the analysis of rail corrugation in eornering by Daniel et al. [8]. The analysis showed clear evidence of roll-slip phenomena, especially on the leading axle.: The sliding oscillation causing wear is mainly a roll-slip oscillation of lateral creer $\mathrm{pe}$ of friction modifiers (FMs) is known to be useful for $\mathrm{r}$ cing corrugation formation, although its effect on the system is still not fully understood [9]. Other effective corrugation mit-igation methods are rail hardening, as well as employing soft railpads in the railway track [6, 10, 11].

The wavelength fixing mechanisms can be excited in different ways. Two main schools of thought have been hitherto developed in scientific literature. The first one considers the excitation of the system in a broad frequency range caused by the longitudinal rail head irregularity, which is assumed to be always present in the form of a random profile with relatively low amplitude. The fluctuation of the friction forces at the same wavelength fixing mechanism frequency leads to differential wear and, in the long run, to corrugation. As such, this the-ory

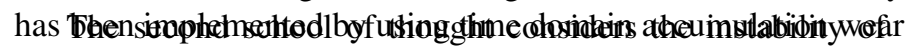
models $[10,12,13]$. the wheel-rail system in specific situations, i.e., self-excited vibrations, as the cause of excitation of wavelength fixing mechanisms. The instability is considered to be caused by the interaction of the wheel and the rail through the contact patch when the creep forces are near saturation, i.e., when the maximum available tangential contact force $\mu \mathrm{N}$ is approached. A first method used to analyze the instability of the system is developed in time domain, and takes into account the roll-slip vibrations between wheel and rail, i.e., the periodic passage of the contact condition from rolling to sliding due to either the periodic variation of the normal contact force between wheel and rail near saturation, or the negative slope of the friction-creepage relationship after saturation [13, 14]. Recently, the instability of the system has been analyzed in the frequency domain by considering the friction-induced dynamic instabilities of the system due to the quasi-saturated friction forces in the neighborhood of a steadystate working condition of the system $[9,15,16]$. This method may be suitable for the prediction of rutting corrugation in short radius curves or in braking or traction sites, and well suitable for performing multiple-cases analyses since frequency domain analyses are generally less time consuming compared to time domain models. Nevertheless, the overall physical mechanism of generation of rail corrugation is still not fully understood.

ABAQUS provides an implemented algorithm for the detection of friction-induced self-excited unstable vibration modes, i.e., complex eigenvalue analysis (CEA). In 2006, AbuBakar and his co-authors $[17,18]$ have used the CEA in combination with a transient dynamic analysis for predicting brake squeal frequencies, and the effect of temperature-dependent friction of the brake stability. In 2007, Liu et al. [19] have performed a parametric analysis of brake squeal by using the same method. In 2009, Nouby et al. [20] haved the CEA with a design of experiment analysis (DOE) in order to perform a parametric analysis of disc brake squeal by using statistical regression techniques. The CEA has been used by Chen et al. to study both rail corrugation and wheel-rail squeal due to self-excited vibrations under saturated creep forces $[9,15,16]$. In these works, a transient dynamic analysis together with the CEA has been used to predict rail corrugation induced by self-excited vibrations.

In this paper, The general aim of this paper is to further expand knowledge on the deployment of the CEA for studying rail corrugation for-mation in short radius curves. A numerical procedure aiming at the prediction of rutting corrugation on short radius curves is carried out through the detection of the self-excited vibrations of the wheelset-track system. The numerical model includes a full track section and a leading wheelset, fully implemented in a Fi-nite Element (FE) environment. Furthermore, a parametric analysis is performed by using the Design of Experiment methodology in order to identify the parameters a significant influence on rail corrugation formation. The overall analysis methodol-ogy adopted in this paper combines multibody simulations for defining correctly the boundary conditions of the FE model and the steady-state position of the wheelset, and FE stability analyses conducted by means of the general-purpose FE software ABAQUS 6.14. 


\section{Wheel-rail contact model}

The FE contact interaction model, which considers a surfacebased approach, is applied to model the wheel-rail contact. The numerical discretization method considers the shape of both master and slave surfaces, such that the contact condition is distributed over the regions near the contacting nodes. The normal interaction between the interacting surfaces is modelled as a hard relationship between the normal pressure and the surfaces overclosure, i.e., the surfaces penetration. This means that no pressure is present when there is no actual contact, while any pressure is possible when actual contact is present. The relationship is enforced numerically by means of a penalty stiff approximation, which can be expressed as:

$$
f_{i}=-\delta_{i} K_{i}
$$

where $f$ is the component of the contact force in normal direction, $\delta$ is the overclosure, $K$ is the contact stiffness and $i$ is the finite element node considered. The tangential contact interaction and simplified Coulomb friction model $(\tau=\mu N)$ for a sufficiently high total contact slip, and an elastic slip $(\tau=\chi \epsilon$, where $\chi$ is the sticking friction and $\epsilon$ is the total slip) for small values of contact slip, in order to take into account the contact sticking creepage. However, since the study assumes quasi-saturation of the contact forces, tangential contact is equivalently modeled by using only the former relation.

\section{Complex Eigenvalue Analysis (CEA)}

The analysis used the official Abaqus User's Manual [21] as technical reference. The complex eigenvalue analysis (CEA) has been widely used mainly as a tool for predicting unstable frequencies in friction-induced vibration problems. The method consists of the computation of system eigenvalues and their corresponding mode shapes which in general are complexvalued functions. The real part of the eigenvalues is deployed as parameter correlated to the stability of the particular mode shape. In general, the system might experience instability because friction forces cause the stiffness matrix to be asymmetric [20].

\subsection{Implementation procedure}

ABAQUS versions 6.4 and later on provide the implementation of the complex eigenvalue analysis for the stability prediction of friction-induced vibration FE models. Generally speaking, the equations of motion of a linear, $n$ degrees of freedom (d.o.f.) mechanical system are:

$$
[M] \underline{\ddot{x}}+[C] \underline{\dot{x}}+[K] \underline{x}=\underline{q}
$$

where $[M],[C]$ and $[K]$ are the mass, damping and structural stiffness matrices of the system (nxn), and $q$ is the vector of the generalized external forces acting on the system. The structural matrices of the system are generally positive definite. In a structural/mechanical FE model, the vector $\underline{x}$ represents all the d.o.f. of the mesh nodes. In the case of the wheel-rail contact problem, the friction forces present in the contact patch are assumed to be the main source of vibration excitation. The vector of external forces $q$, which consists only of the friction forces, can be defined as a function of the displacement vector $\underline{x}$ and the localized contact stiffness matrix:

$$
\underline{q}=\mu\left[K_{f}\right] \underline{x}
$$

where $\left[K_{f}\right]$ is the friction stiffness matrix, generally not symmetric, and $\mu$ is the friction coefficient on the contact patch. The governing equations can hence be rewritter

$$
[M] \underline{\ddot{x}}+[C] \underline{\dot{x}}+\left[[K]-\mu\left[K_{f}\right]\right] \underline{x}=\underline{0}
$$

where $\left[[K]-\mu\left[K_{f}\right]\right]$ is in turn not symmetric. Since the global stiffness matrix is not symmetric, various modes of the system might manifest flutter instability. The characteristic equations of the system can be expressed as:

$$
\left(\lambda^{2}[M]+\lambda[C]+\left[[K]-\mu\left[K_{f}\right]\right]\right) \phi=\underline{0}
$$

where $\lambda$ and $\phi$ are the eigenvalues and the eigenvectors of the system, respectively.

In general, Eq. 3.4 gives complex eigenvalues. It is not trivial to solve an eigenvalue problem with an asymmetric stiffness matrix. Therefore, in order to get a simpler solution of the problem approximation methods are generally used. The most common one is the so-called subspace projection method. In the subspace projection method, the original eigensystem is projected into a subspace spanned by the eigenvectors of the undamped, symmetric system [21]. In absence of the damping and friction terms, Eq. 3.4 reduces to a symmetric problem:

$$
\begin{aligned}
\left(\lambda_{r}^{2}[M]+[K]\right) \underline{\phi_{r}} & =\underline{0} \\
\operatorname{det}\left(\lambda_{r}^{2}[M]+[K]\right) & =\underline{0}
\end{aligned}
$$

where $\phi_{r}$ are the eigenvectors of the reduced system. Eq. $3.5 \mathrm{~b}$ can be easily solved by means of well-implemented iteration methods for the extraction of eigenvalues and the corresponding eigenvectors of a symmetric generalized eigenproblem, e.g., Lanczos or AMS methods. In general, the eigenvalue extraction is stopped before a complete extraction at a user-defined number of eigenvalues $m$. With the reduced solution, all the structural matrices of the original system can be projected into the reduced eigenvector subspace.

$$
\begin{cases}{\left[M^{*}\right]} & =\left[\Phi_{r}\right]^{T}[M]\left[\Phi_{r}\right] \\ {\left[C^{*}\right]} & =\left[\Phi_{r}\right]^{T}[C]\left[\Phi_{r}\right] \\ {\left[K^{*}\right]} & =\left[\Phi_{r}\right]^{T}[K]\left[\Phi_{r}\right]\end{cases}
$$

where $\left[\Phi_{r}\right]$ is the transformation matrix composed of the computed eigenvectors. With the projected structural matrices of the system, the differential equations of motion in the modal subspace can be written as follows:

$$
\left[M^{*}\right] \underline{\ddot{y}}+\left[C^{*}\right] \underline{\dot{y}}+\left[K^{*}\right] \underline{y}=\underline{0}
$$

where $\underline{y}$ is the vector of the modal coordinates, defined as:

$$
\underline{x}=\left[\Phi_{r}\right] \underline{y}
$$


The projected characteristic equations thus become:

$$
\left(\lambda_{p}^{2}\left[M^{*}\right]+\lambda_{p}\left[C^{*}\right]+\left[\left[K^{*}\right]\right]\right) \underline{\phi_{p}}=\underline{0}
$$

where $\phi_{p}$ are the eigenvectors of the projected system.

Eq. 3.4 requires solving a large number of equations, equal to the number of d.o.f. of the FE model. In Eq. 3.9 the are fewer equations, equal to the number of natural eigenmodes used for the subspace projection, thus leading to a faster numerical solution. This equation is then solved using the standard QZ method for generalized non-symmetric eigenproblems.

Finally, the eigenvectors of the original system can be ap $\Omega$ proximated by projecting the obtained eigenvectors in the original $n$ d.o.f. space:

$$
\underline{\phi} \simeq\left\{\underline{\phi_{r 1}}, \underline{\phi_{r 2}}, \ldots, \underline{\phi_{r m}}\right\} \underline{\phi_{p}}
$$

Usually, also in the case of the final complex eigenvalue and eigenvectors, the extraction stops at a user-defined numbe of eigenvalues. The complex eigenvalue $\lambda_{k}$ can be expressed by means of its real and imaginary components $\lambda_{k}=\alpha_{k} \pm i \omega_{k}$. The general solution of the system can he obtained by superimposing the single eigenmodes:

$$
x(t)=\sum_{k=1}^{n} \phi_{k} e^{\lambda_{k} t}=\sum_{k=1}^{n} \phi_{k} e^{\left(\alpha_{k} \pm i \omega_{k}\right) t}
$$

The real part of the eigenvalue is the basis to judge the stability of the system. When the real part of an eigenvalue is larger than zero, the nodal displacement $x(t)$ will increase with time, meaning that the vibration of the system is growing and the system will become unstable according to that particular mode of vibration [15]. A common parameter used to measure the propensity of self-excited vibration occurrence is the definition of an effective damping ratio for every single eigenmode of the system:

$$
\xi_{k}=-2 \frac{\operatorname{Re}\left(\lambda_{k}\right)}{\operatorname{Im}\left(\lambda_{k}\right)}
$$

If the damping ratio is negative, the corresponding eigenmode is supposed to be unstable and has a tendency to induce self-excited vibrations.

The analysis steps needed for performing a complex eigenvalue analysis are hence the following:

- Nonlinear steady-state analysis of the wheelset-track system for applying the suspension forces on the bearings.

- Nonlinear steady-state analysis for imposing the lateral steady-state speed on the wheelset.

- Natural frequency extraction of the system without considering the friction coupling and damping to find the projection subspace.

- Complex eigenvalue extraction of the system that incorporates the effect of the friction coupling and damping.
Ir st $p 2$, the lateral steady-state speed of the wheelset, defined by the wheelset angle of attack $V_{l}=\Omega$ in $\psi$ is imposed through a $* \mathrm{M} \approx \mathrm{m}$, TRANSLATION Cedure and a TYPE=VELOCITY avior to the wheelset FE domain.

\subsection{Corrugation Index}

When studying the formation of corrugation by means of the complex eigenvalue analysis, the effective damping ratio $\xi$ gives the information regarding the propensity of a particular mode of vibration to be self-excited by the dynamic situation. One may also consider the degree of potential slip at the contact patch that can be generated by the unstable modes as correlated with corrugation formation. Wear can be modeled by using Archard's simplified wear theory. This model makes the assumption that the volume of removed material is proportional to the dissipated sliding energy, which in turn is the work done by the friction forces. Therefore, Archard's wear is formed only in the presence of relative creepage. The volume of removed material due to wear is:

$$
V=K \frac{N}{H} \kappa
$$

where $N$ is the normal load, $H$ the hardness of the softer of the two contacting bodies, $\kappa$ is the sliding distance and $K$ the wear coefficient [22]. Chen et al. studied how self-excited vibrations lead to fluctuation of the contact forces through a comparison of a self-excited vibrations analysis and a transient-dynamic analysis in time domain [16]. Hence, self-excited vibrations may influence the contact condition and lead to differential wear.

Sliding distance is always present in the unstable modes of vibration since the contact is assumed quasi-saturated. However, the longer the sliding distance between the contacting bod-ies it is, the bigger will be the removed material due to adhesive wear will be. Therefore, an alternative indicator, the effective corru-gation index $(\gamma)$, which is directly proportional to parameters describing the unstable ility of modes of vibration of the system and their corresponding equivalent sliding distance, is proposed in this study and is defined as:

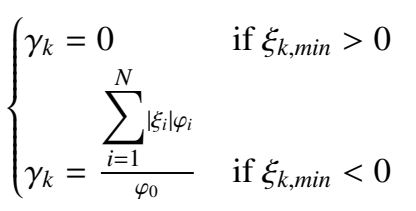

where $k$ is the kth analysis case, $N$ is the number of unstable modes detected in the considered analysis case, $\xi_{i}$ is the $i$ th unstable mode effective damping ratio, $\varphi_{0}$ is a normalization factor equal to $10^{-6}$ and $\varphi_{i}$ is the $i$ th slip factor computed as:

$$
\varphi_{i}=\sqrt{\left(u_{1 w, i}-u_{1 l r, i}\right)^{2}+\left(u_{3 w, i}-u_{3 l r, i}\right)^{2}}
$$

where $u$ is the mean normalized nodal displacement of the unstable mode shape computed in the neighborhood of the contact patch, in the transversal direction (subscript 1) and longitudinal direction (subscript 3 ) for the low wheel (subscript $w$ ) and the low rail (subscript $l r$ ). Only the low wheel and rail are considered in the definition of the slip factor since virtually all 
a)

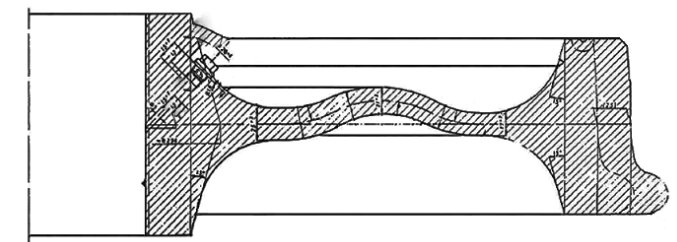

b)

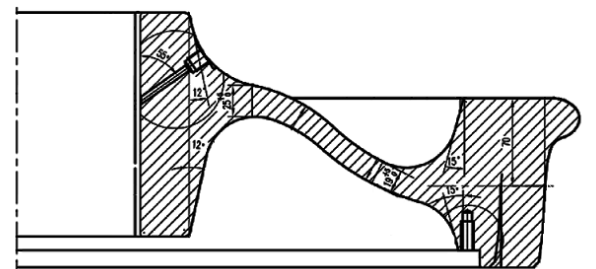

c)

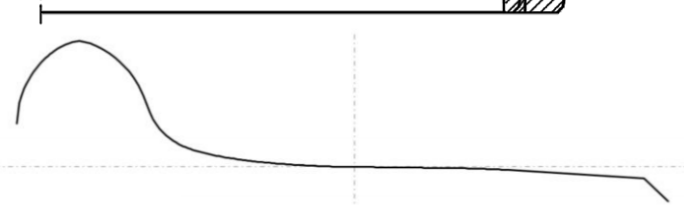

Figure 1: Wheel cross-section (including wheel profile). a) European S-shaped. b) Chinese SFMZ06M1 [15]. c) Wheel profile-EN 13715 S1002/h28/e32.5/6.7\%.

vibration is concentrated in the low side. The nodal mean normalized displacements are computed as an average value of the relative displacement of the mode shape on five different nodes in the contact region. Hence, the effective damping ratio $\xi_{i}$ is used as the instability index of the $i$ th mode, and $\varphi_{i}$ is used as the slip index of the $i$ th mode.

\section{Wheelset-track model}

The wheelset-track model assembly represents a short radius curve section with curve radius $300 \mathrm{~m}$, superelevation or cant $100 \mathrm{~mm}$, rail inclination angle $1 / 20$, sleeper spacing 600 $\mathrm{mm}$. Two different track gauges are considered in the analysis: the standard track gauge $1435 \mathrm{~mm}$ and a wider track gauge $1455 \mathrm{~mm}^{\text {gauge }}$. The rails are modeled considering the track curvature, with a rail curva-ture angle $\beta=l / r$, where $l$ is the rail track length and $r$ is the curve radius. The rails and wheels are divided into geometrical sub-domains in order to apply a refined mesh near the contact patch.

The analysis considers standard rail, sleeper, wheelset geometries, and standard wheel profiles (wheel running surface profiles). The standard UIC60 rail is used in the analysis. Being one of the study parameters, two different wheel cross-sections are considered, as shown in Figure 1. Specifically, a typical European S-shaped wheel cross-section with $920 \mathrm{~mm}$ diameter and a Chinese wheel cross-section (SFMZ06M1) with $840 \mathrm{~mm}$ diameter are considered, the latter being typically used in metro lines and studied by Chen et al. in their corrugation analysis [15]. This geometrical parameter describes simultaneously the effect of multiple sub geometrical parameters, such as wheel diameter, wheel profile, flange ge ometry, radii of curvature, etc. Since this study is primarily concerned about the viability of the complex eigenvalue analysis as a possible method for the

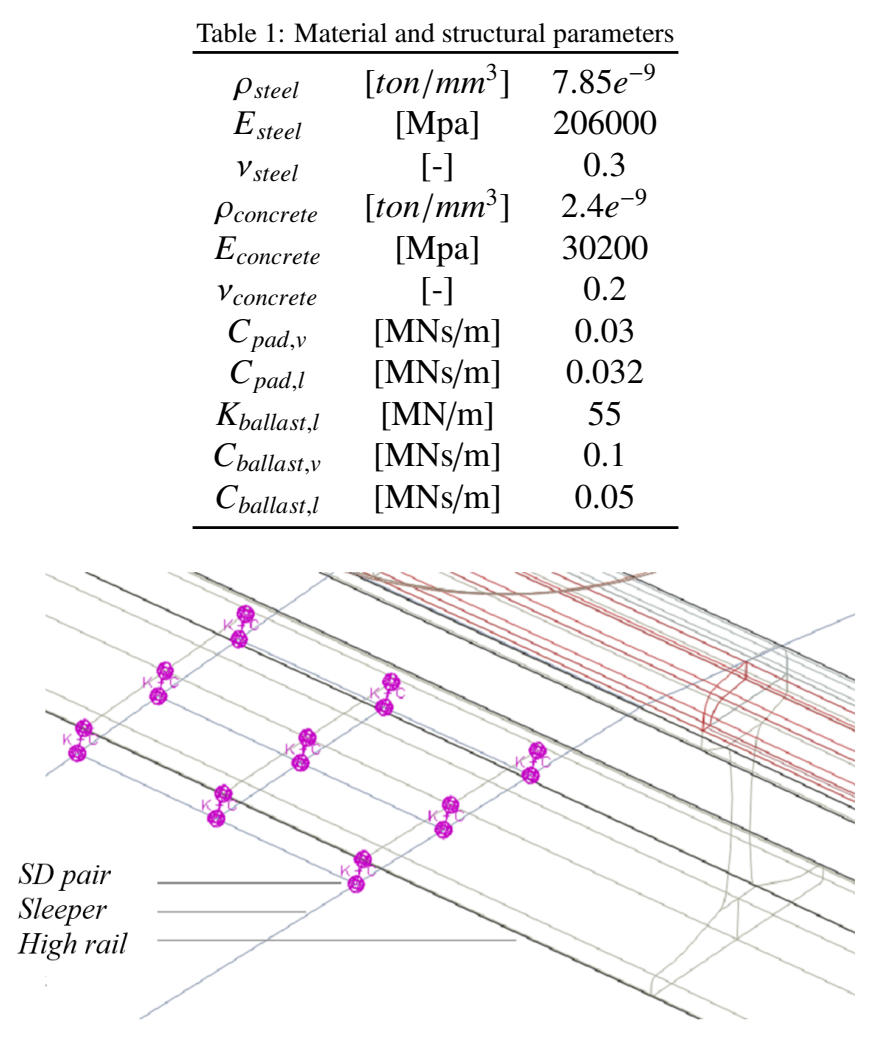

Figure 2: Rail pad spring-dashpot model.

study of rutting corrugation,- and only secondly to get first results about the effects of several parameters by using the abovementioned method, this approach for the study of the effect of wheel geometry has been considered germane with respect to the framework of the present study.

The wheel profile used for both wheel cross-sections is the standard EN 13715 S1002 with flange height of $28 \mathrm{~m} \mathrm{~m}$ and thickness of $32.5 \mathrm{~mm}$. The sleepers used in the studied track are the concrete sleeper JBV60, typically used by the Norwegian Railway Administration in combination with UIC60 rails.

\subsection{Finite element model}

All the materials are assumed to be linear-elastic. The steel materials considered for the wheelset and for the rails are assumed to have the same mechanical properties. Table 1 shows the material parameters and structural param-eters used in the rodel.

2 he rail geometry is modeled in sub-domains for applying a refined mesh near the contact region. Tie couplings are de-fined among the geometry sub-domains in order to constrain the d.o.f. of the surface nodes. In order to apply the primary suspension forces, it is necessary to define two interface nodes by coupling two reference points on the axle surfaces corresponding to the position of the bearings. To this end, kinematic couplings are used. A set of nine spring-dashpot pairs is considered to be sufficient for modeling the rail pad dynamics in the frequency range of interest [0-1000 Hz]. Three spring-damper (SD) elements distributed in the longitudinal direction and three 


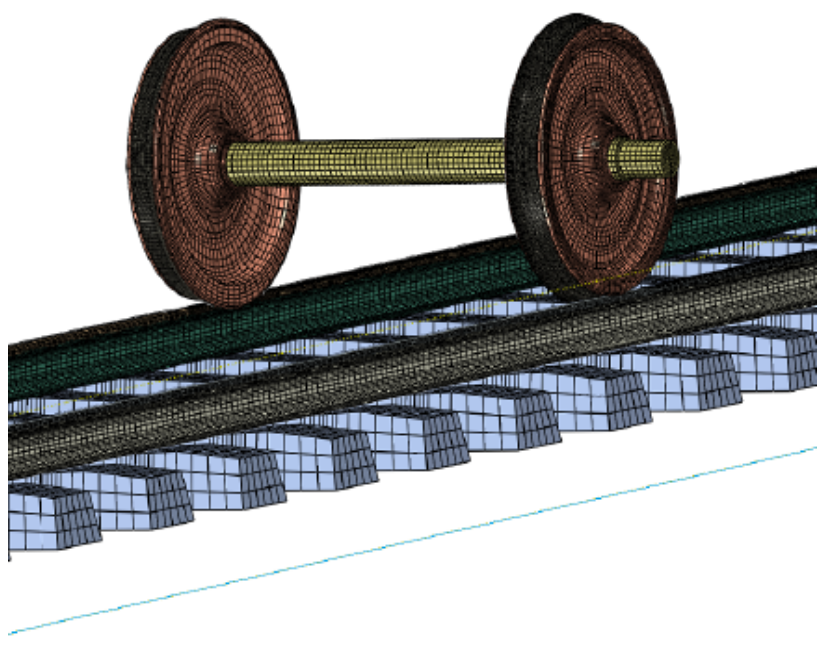

Figure 3: FE meshed model overview.

spring-damper elements distributed in lateral direction for each rail pad are used to account for all the translational and rotational flexibilities of the rail pads [23]. With respect to the modeling solution for railpads and ballast implemented in reference [15], the modeling strategy here proposed uses fewer SD elements, hence contributing to an overall reduction of computational time for the analysis.

Figure 2 depicts the rail pad spring-dashpot area model. In the same way, the ballast is modeled by springs and dashpots by using an Area-SD model approach, as the rails and sleeper seats are covered with multiple discrete linear spring and viscous damper pairs [24]. The mesh density is increased near the contact patch, leading to a global seed size of $35 \mathrm{~mm}$ and a minimum seed size near the contact patch equal to $4 \mathrm{~mm}$. In order to avoid the presence of hourglass unstable zero-energy modes, the 8-node linear brick, incompatible modes elements (C3D8I) are used. Although the best quality of the model can be achieved with quadratic elements, the C3D8I elements generally give a reasonable trade-off between quality, stability and the integration time. The model is composed of circa 316000 C3D8I elements.

Figure 3 depicts an overview of the global meshed FE model used in the analysis. The real structural system has an approximately infinite longitudinal length. However, a too-long track model cannot be used because it would lead to excessive computational effort. A trade-off between rail length, dynamic properties, and computational effort should then be inThe effect of the track longitudinal length on the vertical rail receptance has been analyzed in order to set an optimal track length in the FE model. This has been done by computing the track frequency response function (FRF) in the frequency range [0-2000 Hz] for several track lengths: 4200 $\mathrm{mm}, 7800 \mathrm{~mm}, 11400 \mathrm{~mm}$, and $36000 \mathrm{~mm}$. The track length

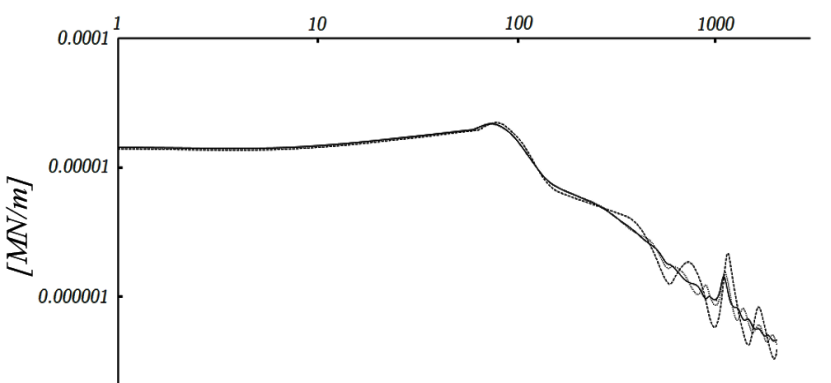

Frequency [Hz]

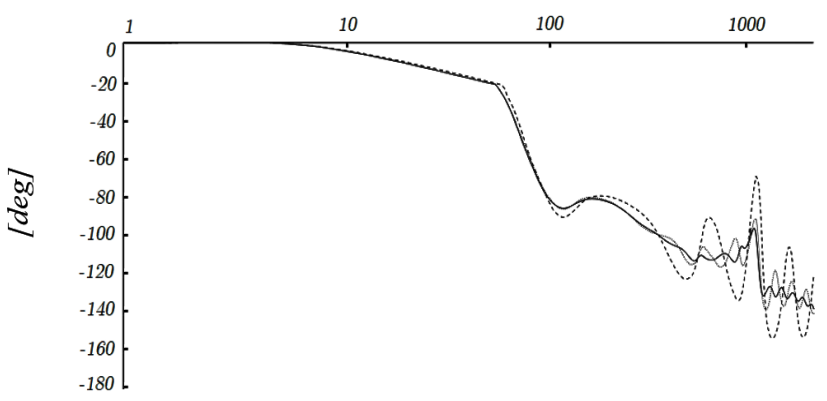

Figure 4: Vertical midspan track receptance functions. a) Magnitude. [N/mm] b) Phase [deg]. (- - ) Rail length: $4200 \mathrm{~mm}$. (…) Rail length: $7800 \mathrm{~mm}$. (-) Rail length: $11400 \mathrm{~mm}$.

of $36000 \mathrm{~mm}$, which is used as a typical track length covering all the interesting vibration modes of the vehicle-track system (also used in reference [15]), led to an excessively large computational time.

Figure 4 depicts the vertical midspan receptance FRF functions and their relative phase functions for the previously defined track lengths. The results shown in Figure 4 are obtained by performing a Steady state modal dynamics simulation with a concentrated unit vertical force acting at the midspan. The effect of the boundary conditions is predominant at $4200 \mathrm{~mm}$ length. An acceptable convergence of the dynamic behavior of the system is obtained for a track length of $11400 \mathrm{~mm}$, as shown in Figure 4, which is hence used as track length of the analysis.

The model proposed in this study shows how, starting from the reference modeling in [15], several modeling strategies can be adopted in order to increase computational efficiency without losing model quality.

\subsection{Implementation of steady-state curving in FE model}

Complex Eigenvalue Analysis is based on a linearization of the equations describing the frictional forces established at wheel/rail interface, through the matrix $\left[K_{f}\right]$, defined in Eq. 3.2. In order to define this matrix, the steady-state curving condition of the vehicle shall be firstly defined, considering the nonlinear effects related to wheel/rail geometry and the saturation effects in the relationship between creepages and creep forces. This task is accomplished by using a multibody system (MBS) model of the entire railway vehicle. This model neglects deformability effects in wheelsets and in the track, and hence it is not suitable for analyzing the formation of corrugation. Yet, it considers in full the non-linearities related to wheel/rail contact in a curve and hence can be used to define the steady-state 


\begin{tabular}{|c|c|}
\hline$F_{h i g h} \bigcap^{[N]}$ & -80000 \\
\hline$F_{\text {low }},{ }_{[N]}$ & -85900 \\
\hline$F_{h i g h, l} \quad[N]$ & 523 \\
\hline$F_{\text {low }, l}$ & 7400 \\
\hline Yaw & -0.008 \\
\hline [rad] & 0.000228 \\
\hline
\end{tabular}

\begin{tabular}{ccr} 
Table 3: MBS results (gauge: $1455 \mathrm{~mm}$ ) \\
\hline$F_{\text {high, }}$ & {$[N]$} & -80000 \\
$F_{\text {low }, v}$ & {$[N]$} & -85900 \\
$F_{\text {high,l }}$ & {$[N]$} & 1710 \\
$F_{\text {low }, l}$ & {$[N]$} & 8610 \\
Yaw & {$[\mathrm{rad}]$} & -0.00812 \\
Roll & {$[\mathrm{rad}]$} & 0.000123 \\
\hline
\end{tabular}

condition of the vehicle as a starting point for the CEA analysis. The evaluation of the steady-state dynamic condition in a curved track of the system is necessary in order to correctly set the FE model for the CE analysis. To this aim, an MBS simulation is performed considering a rigid track model. The necessary input data coming from the simulation are essentially the suspension forces acting on the wheelset bearings. The reference velocity considered is $40 \mathrm{Km} / \mathrm{h}$. The total length of the track in the MBS is $300 \mathrm{~m}$. The track is divided into three distinct sections: a straight section, a transition section, and a curved section. The first section is $24 \mathrm{~m}$ long. The transition section is $51 \mathrm{~m}$ long. The curved section is $225 \mathrm{~m}$ long. In the MBS model, the train is represented by one wagon. The car body rests on two bogies, each one having a leading wheelset and a trailing wheelset. The wheelsets are not motorized and are modeled as rigid bodies. The weights of the car body and the bogie (exces wheelsets) are 47.5 ton and 10 ton, respectively. Two different simulations are performed in relation to the two gauges used in the analysis.

Table 2 and Table 3 show the results for a standard gauge and a wider track gauge, respectively. Subscript $v$ stands for the vertical direction, $l$ for the lateral direction, high for the high rail, and low for the low rail. The vertical forces are positive if pointing upwards while the lateral forces are positive if pointing towards the outer curve side. The positive yaw angle is positive in clockwise direction, the vertical upward direction being the axis reference.

\section{Design of Experiment (DOE) settings}

To find o ut the critical factors a nd e ffective treatments to corrugation, it is essential to investigate how typical parameters of the wheelset and track system might affect the occurrence of cor-rugation in a short radius curve condition in terms of variation of the corrugation index $\gamma$. The analysis is focused on four main factors:

- Rail pad Stiffness. In the literature, rail pad stiffness is
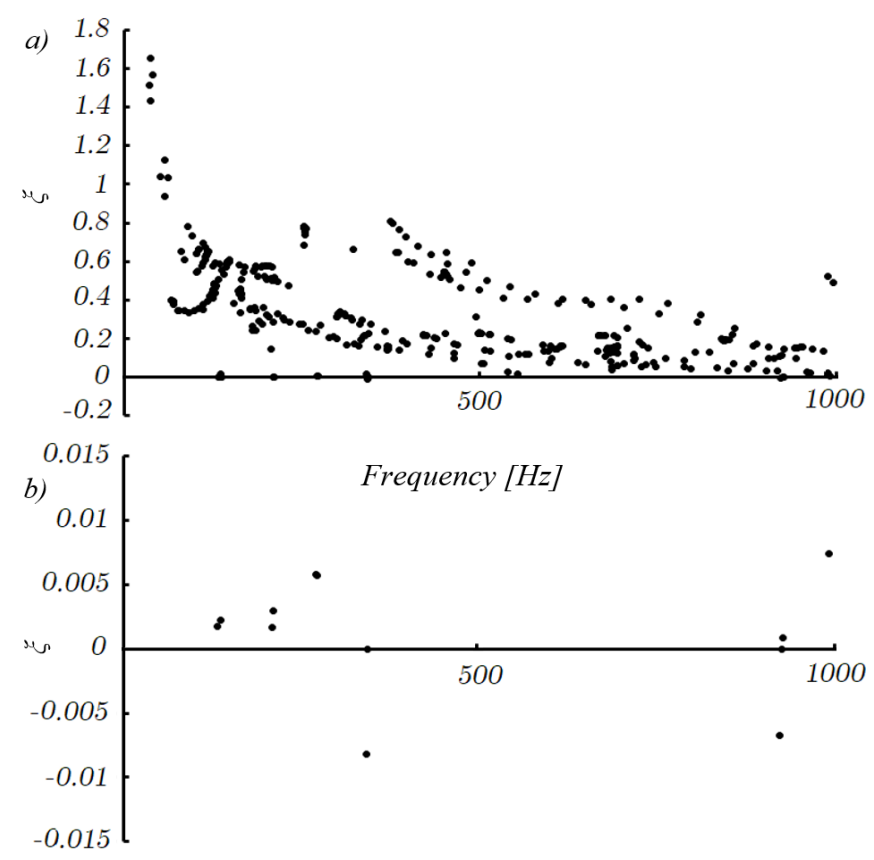

Figure 5: Results in terms of effective damping ratios and their relative frequency. a) General results. b) Emphasis on the unstable eigenmodes.

considered as one of the parameters that can be modified to inhibit corrugation occurrence. A numerical study conducted in 1998 by H. Ilias [10] and the experiment results collected in 2005 by J.Vinolas [11] showed that stiffer rail pads led to higher corrugation growth.

\section{- Vertical Ballast Stiffness.}

- Wheel cross-section. This factor might have a strong impact on the unstable mode shapes properties, hence affecting rail corrugation formation. Two different wheel cross-sections are used.

- Track Gauge. In curved tracks, track gauge is widened in order to get a smooth curving behavior. This factor can change the position of the contact points on the rail and wheel surface, and the friction forces field distribution on the model; hence, it may affect the occurrence of instability.

Table 4 shows the levels of the chosen factors levels. The rail pad constant parameter values and the rail pad stiff-ness levels, as well as the constant parameters of the rail pad, are determined from the study by K. Knothe and S.L. Grassie [25], while the stiffness levels and other constant parameters of the ballast are determined from the studies published by the EUROBALT project [26]. The lateral and longitudinal stiffness of the rail pad and the ballast are considered to be equal. The lateral rail pad stiffness is considered to be circa $20 \%$ of the ver-tical rail pad stiffness $\left(K_{\text {pad }, l} \simeq 0.2 K_{\text {pad,v }}\right)$. The DOE analysis is composed of 36 cases. 
Table 4: Analysis factors

\begin{tabular}{|c|c|c|c|c|}
\hline Factor & & + & 0 & - \\
\hline Vertical Pad Stiffness, $K_{p a d, v}$ & {$[\mathrm{MN} / \mathrm{m}]$} & 600 & 340 & 80 \\
\hline Vertical Ballast Stiffness, $K_{\text {ballast }, v}$ & {$[\mathrm{MN} / \mathrm{m}]$} & 200 & 110 & 20 \\
\hline Wheel cross-section, $w_{c s}$ & {$[-]$} & European & & Chinese \\
\hline Track Gauge, $g$ & {$[\mathrm{~mm}]$} & 1455 & & 1435 \\
\hline
\end{tabular}

\section{Results and discussion}

The results of the analysis are given in terms of complex eigenvalues and complex eigenvectors, i.e., mode shapes, in the frequency range of interest and their associated effective damping ratio. In ABAQUS, the effective damping ratios are collected in the output variable DAMPRATIO. Figure 5a depicts the typical distribution of the complex eigenvalues of the system in terms of effective damping ratio in the frequency range of interest [0-1200 Hz]. The distribution is similar, although not identical, in all design of experiments cases. Here, for the sole purpose of showing the distribution of the effective damping ratios, a single case is considered and it refers to the wider track gauge $1455 \mathrm{~mm}$, a European wheel cross-section, a vertical rail pad stiffness of $340 \mathrm{MN} / \mathrm{m}$, and a vertical ballast stiffness of $20 \mathrm{MN} / \mathrm{m}$. Figure $5 \mathrm{~b}$ zooms in the distribution of the near-zero effective damping ratios. Two negative effective damping ratios are visible in the chart. These two unstable modes may be re-lated to high amplitude vibrations. The first unstable mode (I) is related to an unstable frequency of $340 \mathrm{~Hz}$ and an effective damping ratio of -0.0082 ; the second unstable mode is related to an unstable frequency of $919 \mathrm{~Hz}$ and an effective damping ratio of -0.0067 .

Figure 6 shows the mode shapes associated with the detected unstable modes. A great deal of the vibration at those frequencies affects the wheelset, and in particular the wheel in contact with the low rail. The vibration mostly occurs in lateral direction. In these two modes, sleepers are not participating in the vibration modes. Since these particular vibration displacement distributions are characterized by a considerable difference in normalized displacement between the wheel at the low rail and low rail at the contact patch, they may be also associated with high slip between and therefore to high corrugation rate at the low rail. This numerical result is eengruent with the experimental evidence on rutting corrugation showing a typical corrugation formation at short radius curves on the low rail $[4,5,6,7,8]$. Moreover, the frequency of the most unstable mode is in the typical rutting frequency range. For each DOE case, the negative effective damping ratios with their associated frequency are obtained, as well as the minimum positive effective damping ratio if no unstable mode is detected. Thus, the corrugation index of each case can be obtained.

In the DOE study, the main effects plot shows the average response for a given level of a factor $(+, 0,-)$ for each of the studied factors, whereby the influence of a single factor with respect to the full range of variation of the remaining factors of the analysis is revealed. The trend of the influence of a single factor on the output variable can be thus determined. The inter-

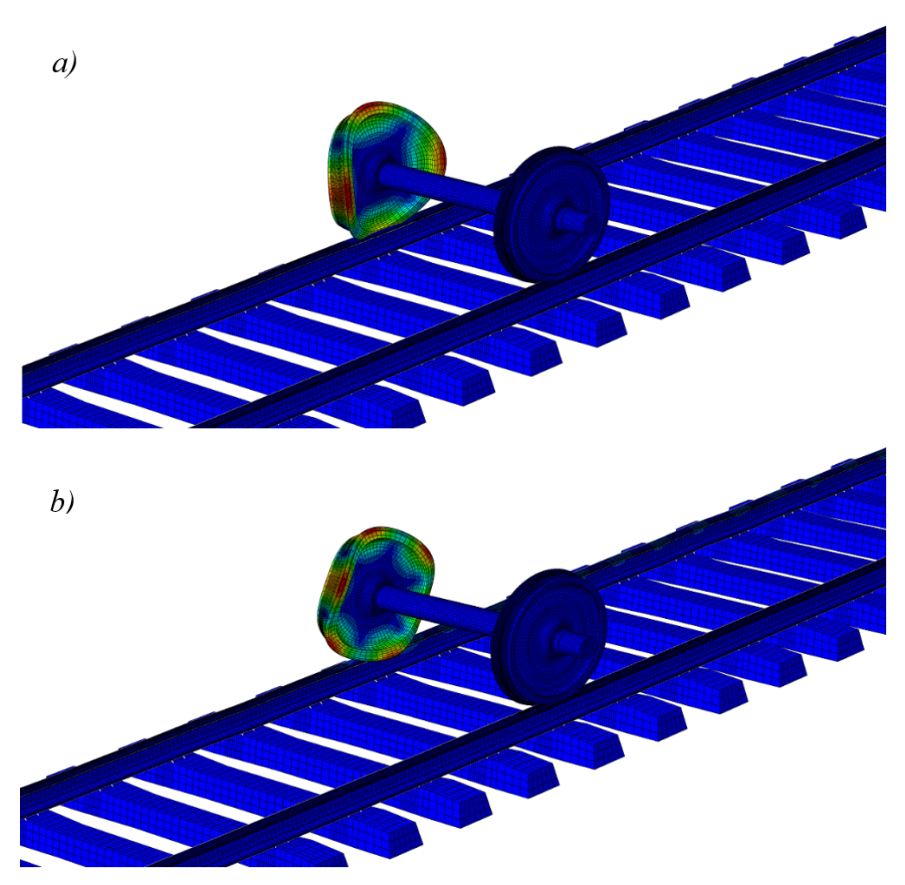

Figure 6: Unstable modeshapes. a) Mode at frequency $340 \mathrm{~Hz}$ and effective damping ratio -0.0082 . b) Mode at frequency $919 \mathrm{~Hz}$ and effective damping ratio -0.0067 .

action plots can also be determined by considering the variation of the average response of a factor due to the level of another factor [27].

In other words, the main effects, $\gamma_{x}$, are computed as:

$$
\begin{array}{rr}
\gamma_{x}=\frac{\left.\sum_{i=1}^{n} \gamma_{i}\right|_{x}}{n} & \text { main effect } \\
\gamma_{x}=\frac{\left.\left.\sum_{i=1}^{n} \gamma_{i}\right|_{x}\right|_{y}}{n} & \text { interaction effect }
\end{array}
$$

where $x$ is the analysis factor level taken into account, $y$ the interaction factor level considered, $\gamma_{i}$ the corrugation index, and $n$ the total number of cases considered.

Figure 7 and 8 show the main effects plots of the four factors and the interaction effect plots with respect to the wheel crosssection. They are acquired by means of the package Minitab and the computation of the Main Effects Plot under Factorial Design [28]. The two-level factors are interpolated through linear functions, while the three-level factors are interpolated 

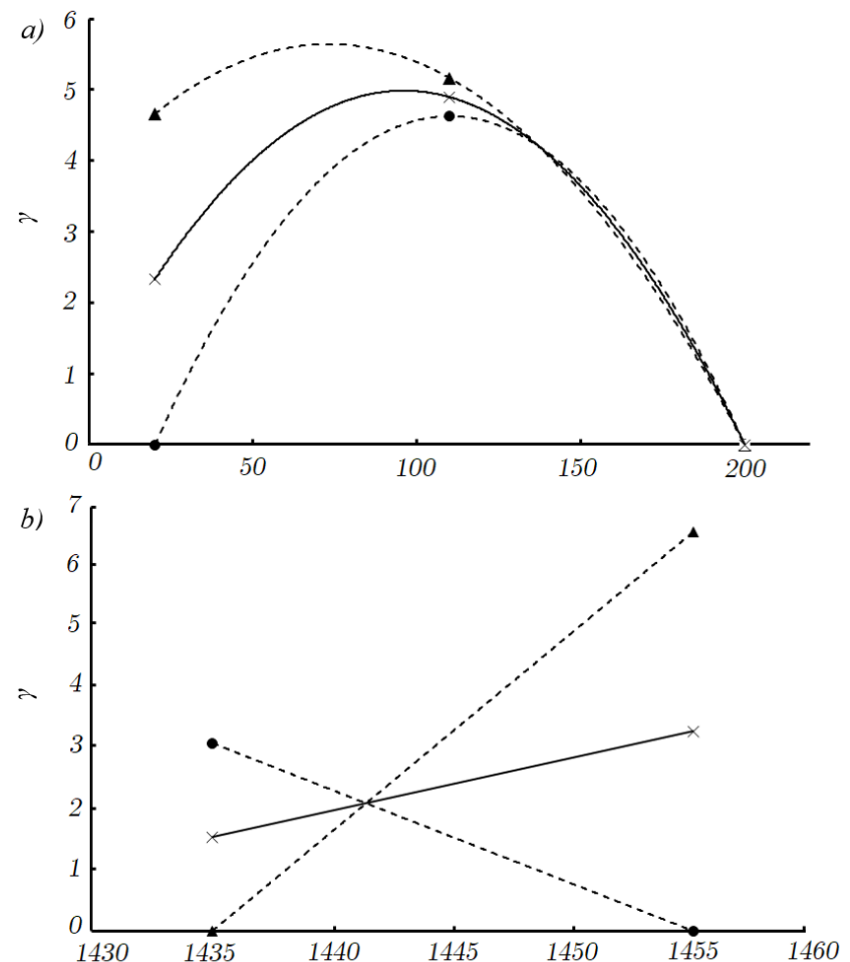

Figure 7: Effects plots and interaction plots with respect to the wheel crosssection. a) Vertical ballast stiffness $[\mathrm{MN} / \mathrm{m}]$. b) Gauge $[\mathrm{mm}]$. (—): Main effects. (- - -•): Wheel cross-section: Chinese. (- - - $\mathbf{\Delta})$ : Wheel cross-section: European.
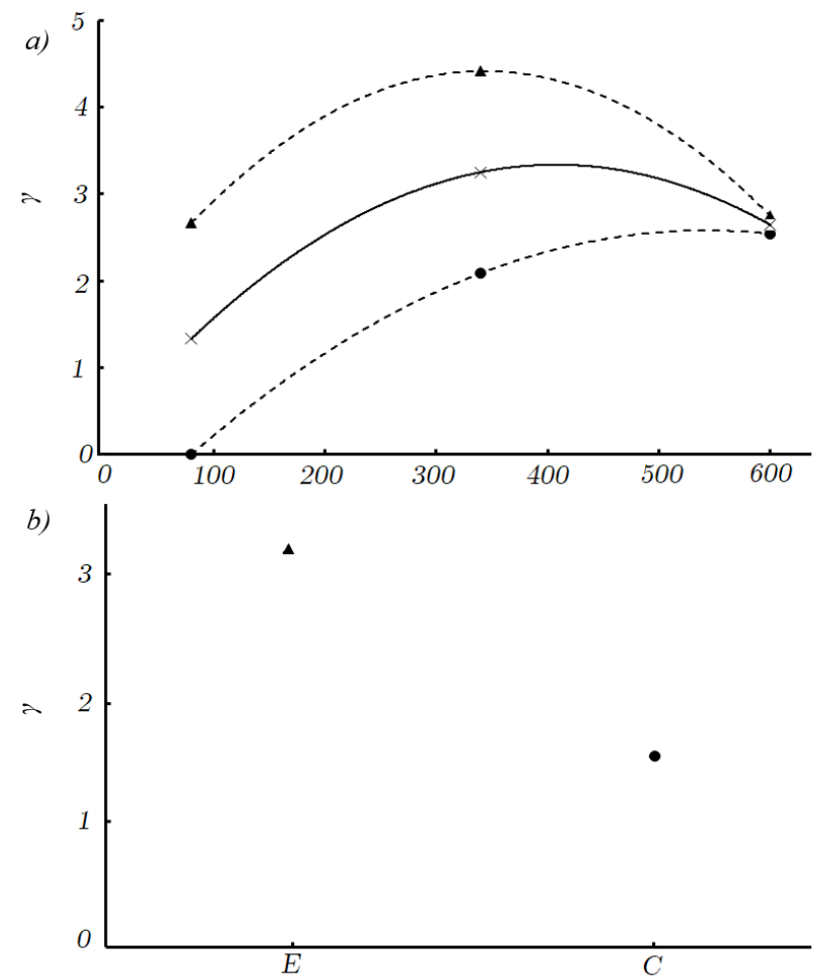

Figure 8: Effects plots and interaction plots with respect to the wheel crosssection. a) Vertical railpad stiffness $[\mathrm{MN} / \mathrm{m}]$. b) Wheel cross-section [-].

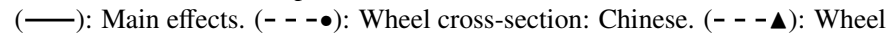
cross-section: European. through quadratic functions. From these results, the following conclusions can be drawn:

- The corrugation index shows its maximum level for a medium vertical ballast stiffness value, i.e., $110 \mathrm{MN} / \mathrm{m}$.

- Evidence of a trend towards lower values of corrugation index for low rail pad stiffness values, e.g., $80 \mathrm{MN} / \mathrm{m}$; This result is in accordance with most of the literature on rail corrugation in that the use of soft rail pads may be a mitigating factor $[4,5,10,11]$.

Figure $7 \mathrm{~b}$ shows the interaction effect of the track gauge and the wheel cross-section, and its strong influence on the corrugation index. Hence, the effect of the track gauge on the corrugation index greatly depends on the wheel cross-section. For instance, in the case of European wheel cross-section, the system is affected by unstable modes only for wider track gauges, specifically for a track gauge of $1455 \mathrm{~mm}$.

The Pareto chart of effects gives a graphical representation of the importance of a single factor or the interaction effects of multiple factors by showing their absolute effect. The effect of each factor and the interaction effect of two or more factors are defined by considering the Statistical Analysis of the t-test, also known as Signal-to-Noise test. This test computes the ratio between the difference of the means of the response and the standard error of the difference. The difference between the maximum and the minimum means can be calculated as:

- for two-levels factors:

$$
X_{i}=\left(a v_{i,+}, a v_{i,-}\right)_{\text {max }}-\left(a v_{i,+}, a v_{i,-}\right)_{\text {min }}
$$

- for three-levels factors:

$$
X_{i}=\left(a v_{i,+}, a v_{i, 0}, a v_{i,-}\right)_{\max }-\left(a v_{i,+}, a v_{i, 0}, a v_{i,-}\right)_{\min }
$$

where $a v_{i,+}, a v_{i, 0}$ and $a v_{i,-}$ are the average responses of the $i$ th factor at the,$+ O$ and - levels. The $t$ value, which is also referred to as the standardized effect, is defined as [29]:

$$
t=\frac{X_{i}}{\sqrt{\frac{\sigma_{i, \max }^{2}}{n_{i, \text { max }}}+\frac{\sigma_{i, \text { min }}^{2}}{n_{i, \text { min }}}}}
$$

where $\sigma_{i, \max }$ is the variance of the response of the $i$ th factor for the maximum case, $\sigma_{i, \min }$ is the variance of the response of the $i$ th factor for the minimum case, $n_{i, \text { max }}$ is the number of samples for the maximum case and $n_{i, \min }$ is the number of samples for the minimum case. A level of significance $(\alpha)$ of 0.05 is used.

Figure 9 depicts the Pareto-chart of standardized-effects. The Figure is acquired through the software package Minitab and the computation of the Pareto Chart of the Standardized Effects under Factorial Design. The terms A, B, C, AB, etc. are the factors considered in the analysis that may contribute in the response, here parametrized with the corrugation index. They can be either main effects, e.g., A, or interaction effects, e.g., $\mathrm{AB}$. The dashed line represents the significance level equivalent 


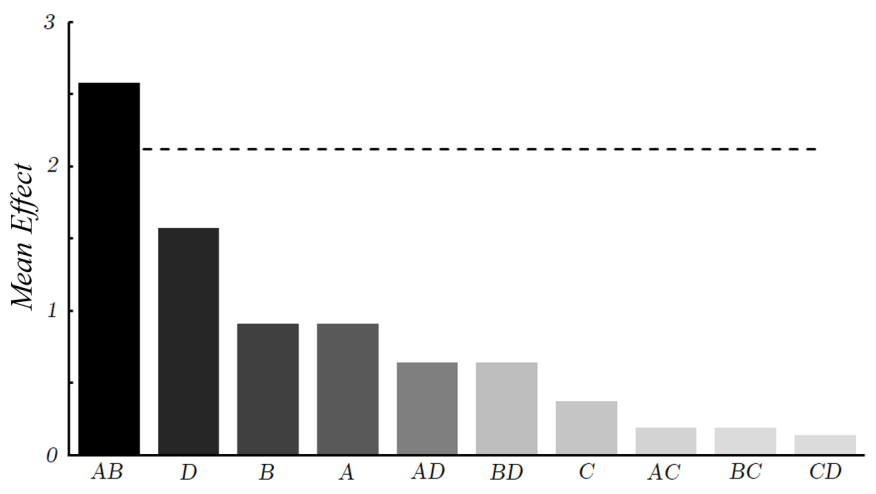

Figure 9: Pareto general chart of the main and interaction effects on $\gamma$. A: Rail Gauge. B: Wheel cross-section. C: Vertical Railpad stiffness. D: Vertical Ballast stiffness.

to an alpha level of 0.05 . The strong influence of the inte effect between the track gauge and the wheel cross-section on the corrugation index is here clearly depicted.

\subsection{Effect of friction coefficient and rail pad damping}

In order to analyze the effect of factors not taken into account in the design of experiments on the corrugation index, a single DOE case taken from the analysis is here considered as a study model. The same DOE case of Figures 5 and 6 has been considered. The additional parameters that have been analyzed are the friction coefficient and the rail pad vertical damping.
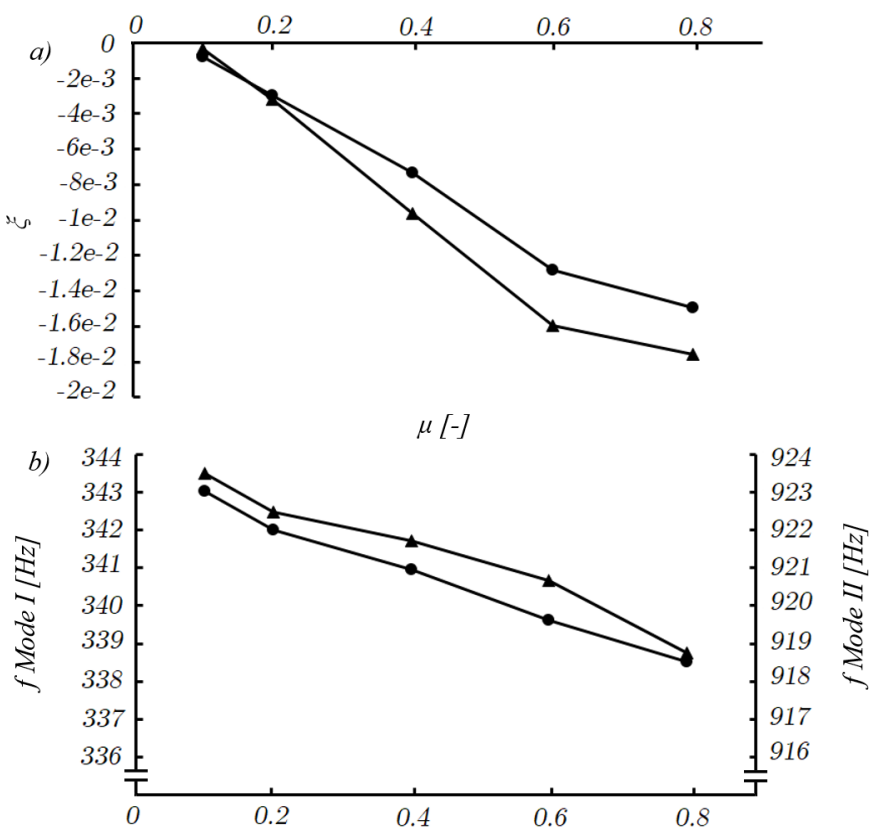

Figure 10: a) Effect of the friction coefficient $\mu$ on the effective damping ratio. $\bullet$ I mode. $\boldsymbol{\Delta}$ : II mode. b) Effect of the friction coefficient $\mu$ on the frequency of unstable vibration. $\bullet$ : I mode. $\boldsymbol{\Delta}$ : II mode.

Figure 10a shows the effect of the friction coefficient on the effective damping ratio of the unstable modes. The effective damping ratio absolute value-which could be related to

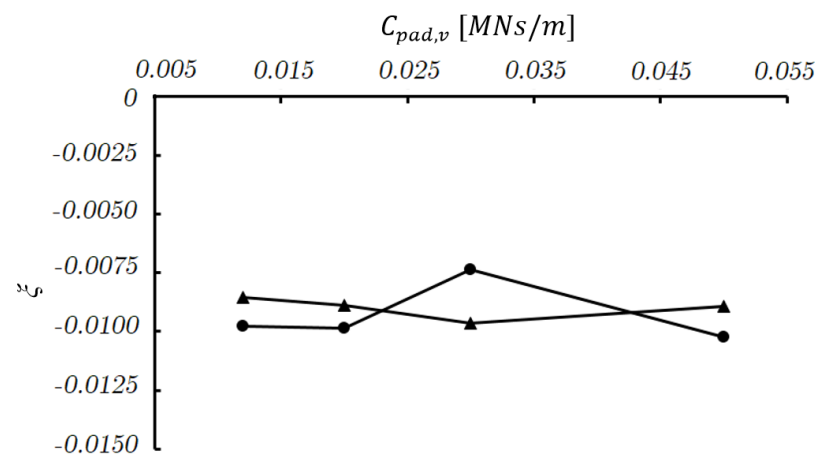

Figure 11: Effect of the vertical railpad damping on the effective damping ratio. $\bullet$ : I mode. $\mathbf{\Delta}$ : II mode.

the rate of growth of corrugation-increases linearly with respect to the friction coefficient, but the sign of the real part is not influenced. Figure $10 \mathrm{~b}$ shows the effect of the friction coefficient on the frequency of the unstable modes. The relationship, by zooming in the results, is characterized by a linear shift of the frequencies of both unstable modes at a lower range for higher friction coefficients. However, the relative shift of the frequencies is too small to conclude that small that one can conclude that the friction coefficient has little influence on the frequency of the unstable modes. Experimental evidence from field observation of cor-rugation growth rate and wavelength shifts with respect to the friction coefficient would be in any case $u$ useful for evaluating the validity of the method.

The same procedure has been followed to analyze the effect of the vertical rail pad damping on self-excited vibrations occurrence. Figure 11 shows the effect of the vertical rail pad damping on the effective damping ratio of the unstable modes. There is no clear evidence of correlation. There might still be interaction effects between this factor and other factors. However, this aspect has not been considered in the present paper.

\section{Conclusions and recommendations}

\subsection{Conclusions}

This paper focused on the still open research question of rail corrugation. Friction-induced self-excited vibration is one of the mechanisms proposed in the past as the cause of corrugation, but was devoted less attention in past research work compared to other proposed explanations of this phenomenon. This work aimed at expanding the knowledge on this less-researched theory/mechanism. A 3D finite e lement m odel, considering the wheelset and the track, was developed in ABAQUS. The effective damping ratio of the coupled wheelset-track system, which was obtained through the complex eigenvalue analysis, was used to calculate the corrugation index. The results obtained by this model are in good qualitative agreement with previous theoretical studies and with experimental evidence on rutting corrugation coming from field observation.

The unstable modes detected in the analysis always show the largest vibration displacement at the wheel running over the 
low rail. This is in good agreement with the evidence that rutting corrugation in short radius curves is almost always formed at the low rail. Furthermore, most of the unstable cases have the most unstable mode at a typical rutting fixing frequency range-between 340 and $495 \mathrm{~Hz}$. The design of experiment predicts a lower corrugation index for softer rail pads, which is in agreement with another research finding from the literature. Therefore, the numerical results collected appear to support the viability of the complex eigenvalue analysis for the study of rutting corrugation.

The analysis of the effect of the friction coefficient on the wheelset stability showed that for higher friction coefficient values, the effective damping ratio associated with the unstable vibration increases in magnitude while the frequency of unstable vibration shifts towards lower values. The design of experiments analysis also showed a strong interaction effect of the track gauge and the wheel cross-section on the corrugation index. The gauge widening applied at short radius curves may led to instability of the wheelset-track system when using the European wheel cross-section, but not with the Chinese wheel cross-section considered in the study. However, without applying the gauge widening, instability of the wheelset-track system may occurred when using the Chinese wheel cross-section, but not with the European wheel cross-section. A thorough physical explanation is not easy to provide straight away, even though the phenomenon is likely to be associated with the interaction between the wheelset resonances, affected by the cross-section of the wheel, and the Rolling Radius Difference (RRD) func-tion resulting from the coupling of the wheel and rail transver-sal profiles, affected by the rail gauge and determining the dis-tribution of the friction forces. A physical justification of this effect will be the subject of further research. Hence, within the assumptions laid out in the proposed method, wheel cross-section and track gauge may be jointly considered as potential inhibitor parameters of rutting corrugation formation in short radius curves.

\subsection{Recommendations}

Two main recommendations for the extension of the present study are laid out: firstly, a study of the wheel cross-section morphology influence on the instability of the wheelset-track system should be performed, with the general aim of developing an optimized wheel cross-section with respect to rutting corrugation formation in short radius curves. Secondly, a study addressing the influence of wheel profile wear on the system instability should be investigated.

\section{Acknowledgments}

The authors gratefully thank Prof. G.X. Chen of the Tribology Research Institute, State Key Laboratory of Traction Power, Southwest Jiaotong University for valuable comments and discussions.

\section{References}

[1] J. Alias, "Characteristics of wave formation in rails," Rail International, no. $11,1986$.

[2] J. K. Oostermeijer, "Review on short pitch rail corrugation studies," Wear, vol. 265 , no. 9, pp. $1231-1237,2008$

[3] E. G. Vadillo, J. Tarrago, G. G. Zubiaurre, and C. A. Duque, "Effect of sleeper distance on rail corrugation," Wear, vol. 217, no. 1, pp. 140 - 145, 1998.

[4] S.L. Grassie, J. A. Elkins, "Rail corrugation on north american transit systems," Vehicle System Dynamics, no. 28, p. 517, 1998.

[5] S.L. Grassie, J. Kalousek, "Rail corrugation: characteristics, causes and treatments," Proceedings of the Institution of Mechanical Engineers, Part F: Journal of Rail and Rapid Transit, vol. 207, no. 1, pp. 57-68, 1993.

[6] S.L. Grassie, "Rail corrugation: Characteristics, causes, and treatments," Proceedings of the Institution of Mechanical Engineers, Part F: Journal of Rail and Rapid Transit, vol. 223, no. 6, pp. 581-596, 2009.

[7] M. Ishida, T. Moto, and M. Takikawa, "The effect of lateral creepage force on rail corrugation on low rail at sharp curves," Wear, vol. 253, Issues 1-2, pp. 172-177, 2002.

[8] W.J.T. Daniel, R.J. Horwood, P.A. Meehan, and N. Wheatley, “Analysis of rail corrugation in cornering," Wear, vol. 265, Issues 9-10, pp. 1183$1192,2008$.

[9] G. Chen, Z. Zhou, H. Ouyang, X. Jin, M. Zhu, and Q. Liu, "A finite element study on rail corrugation based on saturated creep force-induced self-excited vibration of a wheelset track system," Journal of Sound and Vibration, vol. 329, no. 22, pp. 4643 - 4655, 2010.

[10] H. Ilias, "The influence of railpad stiffness on wheelset/track interaction and corrugation growth," Journal of Sound and Vibration, vol. 227, no. 5, pp. $935-948,1999$.

[11] J. Egana, J. Vinolas, and M. Seco, "Investigation of the influence of rail pad stiffness on rail corrugation on a transit system," Wear, vol. 261, no. 2, pp. $216-224,2006$.

[12] P. Meehan, W. Daniel, and T. Campey, "Prediction of the growth of weartype rail corrugation," Wear, vol. 258, no. 7, pp. 1001 - 1013, 2005.

[13] Y. Sun, S. Simson, "Wagontrack modelling and parametric study on rail corrugation initiation due to wheel stick-slip process on curved track," Wear, vol. 265, no. 9, pp. $1193-1201,2008$

[14] A. Matsumoto, Y. Sato, H. Ono, M. Tanimoto, Y. Oka, and E. Miyauchi, "Formation mechanism and countermeasures of rail corrugation on curved track," Wear, vol. 253, no. 1, pp. 178 - 184, 2002.

[15] X. L. Cui, G. X. Chen, H. G. Yang, Q. Zhang, H. Ouyang, and M. H. Zhu, "Study on rail corrugation of a metro tangential track with cologne-egg type fasteners," Vehicle System Dynamics, vol. 54, no. 3, pp. 353-369, 2016.

[16] W. Qian, G. Chen, H. Ouyang, M. Zhu, W. Zhang, and Z. Zhou, "A transient dynamic study of the self-excited vibration of a railway wheelsettrack system induced by saturated creep forces," Vehicle System Dynamics, vol. 52, no. 9, pp. 1115-1138, 2014.

[17] A. R. AbuBakar, H. Ouyang, "Complex eigenvalue analysis and dynamic transient analysis in predicting disc brake squeal," International Journal of Vehicle Noise and Vibration, vol. 2, no. 2, pp. 143-155, 2006.

[18] A. R. AbuBakar, M. K. A. Hamid, A. Dzakaria, B. A. Ghani, and M. Mohamad, "Stability analysis of disc brake squeal considering temperature effect," Jurnal Mekanikal, vol. 22, pp. 26-38, 2006.

[19] P. Liu, H. Zheng, C. Cai, Y. Wang, C. Lu, K. Ang, and G. Liu, "Analysis of disc brake squeal using the complex eigenvalue method," Applied acoustics, vol. 68, no. 6, pp. 603-615, 2007.

[20] M. Nouby, D. Mathivanan, and K. Srinivasan, "A combined approach of complex eigenvalue analysis and design of experiments (doe) to study disc brake squeal," International Journal of Engineering, Science and Technology, vol. 1, no. 1, pp. 254-271, 2009.

[21] A. Inc., “Abaqus analysis user's manual, version 6.14,” 2016.

[22] J.F. Archard, W. Hirst, "The wear of metals under unlubricated conditions," Proc. Roy. Soc. A 236 387-410, 1956

[23] X.P. Cai, E. Kassa, "Detection of Acceleration Sensitive Areas of Rail using a Dynamic Analysis," Third International Conference on Railway Technology, Cagliari, Sardinia, Italy, Paper 275, 2016.

[24] M. Oregui, Z. Li, and R. Dollevoet, "An investigation into the modeling of railway fastening," International Journal of Mechanical Sciences, vol. 92, pp. 1-11, 2015. 
[25] K. Knothe, S. Grassie, "Modelling of railway track and vehicle/track interaction at high frequencies," Vehicle system dynamics, vol. 22, no. 3-4, pp. 209-262, 1993.

[26] J. Huille, "Eurobalt will tap the unexploited potential of ballasted track," Railway Gazette International, 1994.

[27] S. Ghosh, C. R. Rao, "Handbook of Statistics 13: Design and Analysis of Experiments," Elsevier Science Pub Co, 1996.

[28] M. Inc., "Minitab 17 user's manual," 2016.

[29] B. Derrick, D. Toher, P. White, "Why Welch's test is Type I error robust," 2016. 